\section{CONSIDERACIONES PARA PROGRAMAR LA REGENERACIÓN DE CONDOMINIOS SOCIALES EN ALTURA. ESTUDIO COMPARADO DE TRES CASOS EN CHILE}

Francisco Chateau ${ }^{1}$, Cristian Schmitt ${ }^{2}$, Alejandra Rasse ${ }^{3}$ y Paula Martínez ${ }^{4}$

\section{Resumen}

El Programa de Regeneración de Conjuntos Habitacionales (PRCH) del MINVU ha venido a hacerse cargo de la necesidad urgente de reducir el déficit cualitativo de la vivienda social y mejorar los edificios de condominios en altura. A partir del aprendizaje de las primeras experiencias se elaboró un modelo de gestión donde se pueden constatar una serie de problemáticas derivadas de los largos plazos de ejecución de estos proyectos. El presente estudio identifica las dimensiones de la gestión, diseño e implementación de procesos que permitirían reducir sus plazos de aplicación,

\section{CONSIDERATIONS FOR PROGRAMMING THE REHABILITATION OF HIGH-RISE SOCIAL CONDOMINIUMS. COMPARATIVE STUDY OF THREE CASES IN CHILE}

\section{Abstract}

The Chilean Ministry of Housing and Urban Planning (MINVU) Housing Complex Regeneration Program was established in response to the urgent need of reducing the qualitative deficit in social housing and to upgrade high-rise housing condominiums. Based on early experiences, a management model was outlined that allowed to identify a series of problems derived from these projects' lengthy execution schedules. This study identifies dimensions in the management, design and implementation of the processes that will allow to reduce their implementation times, by means of a comparative 
por medio de un análisis comparativo de tres casos de estudio en desarrollo. En la etapa de diagnóstico, se recomienda la reorganización de los estudios requeridos; y en la etapa de construcción, reconsiderar la tecnología aplicada a los sistemas constructivos, que posee un impacto relevante en los plazos de ejecución.

PALABRAS CLAVE: PROGRAMA DE REGENERACIÓN; REHABILITACIÓN; VIVIENDA COLECTIVA EN ALTURA; BLOQUES TIPO C; METODOLOGÍA DE INTERVENCIÓN; REGENERACIÓN URBANA; REGENERACIÓN DE BARRIOS; RETROFIT.

Recibido: 2020-03-31

Aceptado: 2020-09-28 analysis of three study cases under development. At the diagnostics stage, the reorganization of the required studies is recommended. At the mobility and construction stage, the technology applied to construction systems has a relevant impact on execution schedules.

\section{KEYWORDS: HOUSING COMPLEX REGENERATION PROGRAM;REHABILITATION;HIGH-RISEHOUSING COMPLEX; C-TYPE BLOCKS; INTERVENTION METHODOLOGY; URBAN REGENERATION; NEIGHBORHOOD REGENERATION; RETROFIT.}

Received: 2020-03-31

Accepted: 2020-09-28
1 Facultad de Arquitectura, Diseño y Estudios Urbanos, Pontificia Universidad Católica de Chile, Chile, https://orcid. org/0000-0002-1082-8851. Correo electrónico: fchateau@ uc.cl

2 Facultad de Arquitectura, Diseño y Estudios Urbanos, Pontificia Universidad Católica de Chile, Chile, https://orcid.org/00000002-8038-5750.Correo electrónico: cschmitt@uc.cl
3 Centro de Desarrollo Urbano Sustentable, Pontificia Universidad Católica de Chile, Chile, https://orcid.org/00000003-0625-8021. Correo electrónico: arasse@uc.cl

4 Pontificia Universidad Católica de Chile, Chile, https:// orcid.org/0000-0001-6973-1432. Correo electrónico: paulamartinez@uc.cl 


\section{Introducción}

Durante las últimas cinco décadas, las ciudades chilenas casi han triplicado su población. La demanda de vivienda sigue creciendo y se calcula que, para el año 2050, cerca de 18 millones (90\%) de chilenos vivirán en áreas urbanas (United Nations, 2015), estimándose que se requerirá construir aproximadamente un millón de nuevas viviendas en las ciudades del país, destinadas fundamentalmente a familias de bajos ingresos.

Con el objetivo de responder a este crecimiento, el Estado ha construido a lo largo de las últimas décadas más de 340 mil departamentos en condominios sociales en altura (Ministerio de Vivienda y Urbanismo, 2014a). La implementación de este modelo de vivienda social ha permitido reducir significativamente el déficit cuantitativo en el país, mas ha consolidado un parque de viviendas colectiva en altura con serias deficiencias cualitativas - superficie insuficiente, envolvente de mala calidad, ausencia de aislación acústica e instalaciones en mal estado-, localizado generalmente en la periferia de las zonas urbanas y con ausencia generalizada de equipamientos, infraestructura y áreas verdes. (IDIEM, 2014).

Actualmente, el Estado de Chile cuenta con diversos programas y herramientas administrativas para implementar proyectos de rehabilitación y regeneración de condominios sociales en altura, sin embargo la dificultad en la gestión comunitaria, la atomización en la propiedad del suelo y las dificultades legales que esto implica, así como las dificultades logísticas y tecnológicas asociadas a la intervención en conjuntos habitacionales habitados, han dificultado el desarrollo masivo de este tipo de proyectos y han hecho necesario sistematizar, simplificar y reducir costos en las diferentes etapas del proceso con el objetivo de promover su implementación en una escala masiva.

\section{El problema de la regeneración de conjuntos de vivienda en altura: una perspectiva chilena e internacional}

Abordar el problema de la vivienda colectiva en altura en Chile requiere de una aproximación multidimensional y una estrategia de resolución multisectorial. En él se superponen cuestiones tan diversas como la superficie de los departamentos - que en su gran mayoría no alcanza el mínimo establecido por la actual normativa chilena-, la mala calidad de la envolvente —incapaz de proveer la aislación y ventilación necesaria para las estaciones de invierno y verano-, la segregación urbana y la creación de guetos en la periferia de 


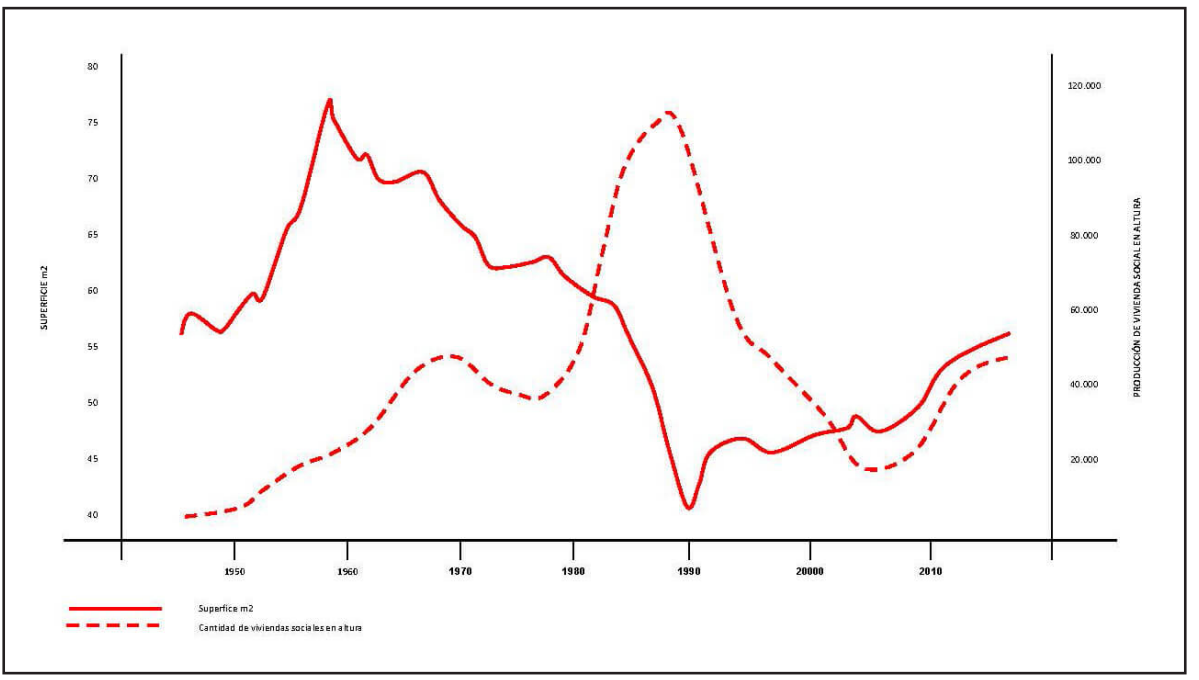

Fuente: Elaboración propia basada en Ministerio de Vivienda y Urbanismo, 2014b.

la ciudad —con la consiguiente falta de equipamientos, servicios e inequidad en las oportunidades de accesos a los bienes de uso público presentes en la ciudad- o la situación legal de las copropiedades — que en muchos casos presentan ampliaciones ilegales $\mathrm{u}$ ocupaciones ilegales de terreno en el primer piso- dificultando la ejecución de programas de regeneración o rehabilitación.

Rodríguez y Sugranyes (2005) han identificado, desde hace más de una década, la insatisfacción de los usuarios de viviendas obtenidas mediante subsidio del Estado, situación que también confirman otros estudios más recientes (Ibañez, 2015). El tamaño insuficiente de los departamentos - la mayoría de los localizados en los denominados bloques " $C$ " tiene una superficie que varía entre los $36 \mathrm{~m} 2$ y los $39 \mathrm{~m} 2$ - ha forzado a los vecinos a realizar ampliaciones informales de mala calidad, altamente riesgosas y sin ninguna posibilidad de regularizarse. Y en cuanto a los espacios comunes, se evidencian disputas por su control; desde las apropiaciones en primer 
FIGURA 2. LEVANTAMIENTO DE CONDOMINIOS SOCIALES EN ALTURA EN LA REGIÓN METROPOLITANA (EN ROJO LOS CONJUNTOS DE BLOQUES C). CATASTRO REALIZADO POR CECILIA CAMARGO, BENJAMÍN CASTRO, SALVADOR ESCOBAR, FERNANDA EZEQUIEL, SIMÓN HERRERA, CHARLES LOUIS LAVANCHY, GIORGIO GANDINO, CONSUELO SAGAL Y VALENTINA SOTO.

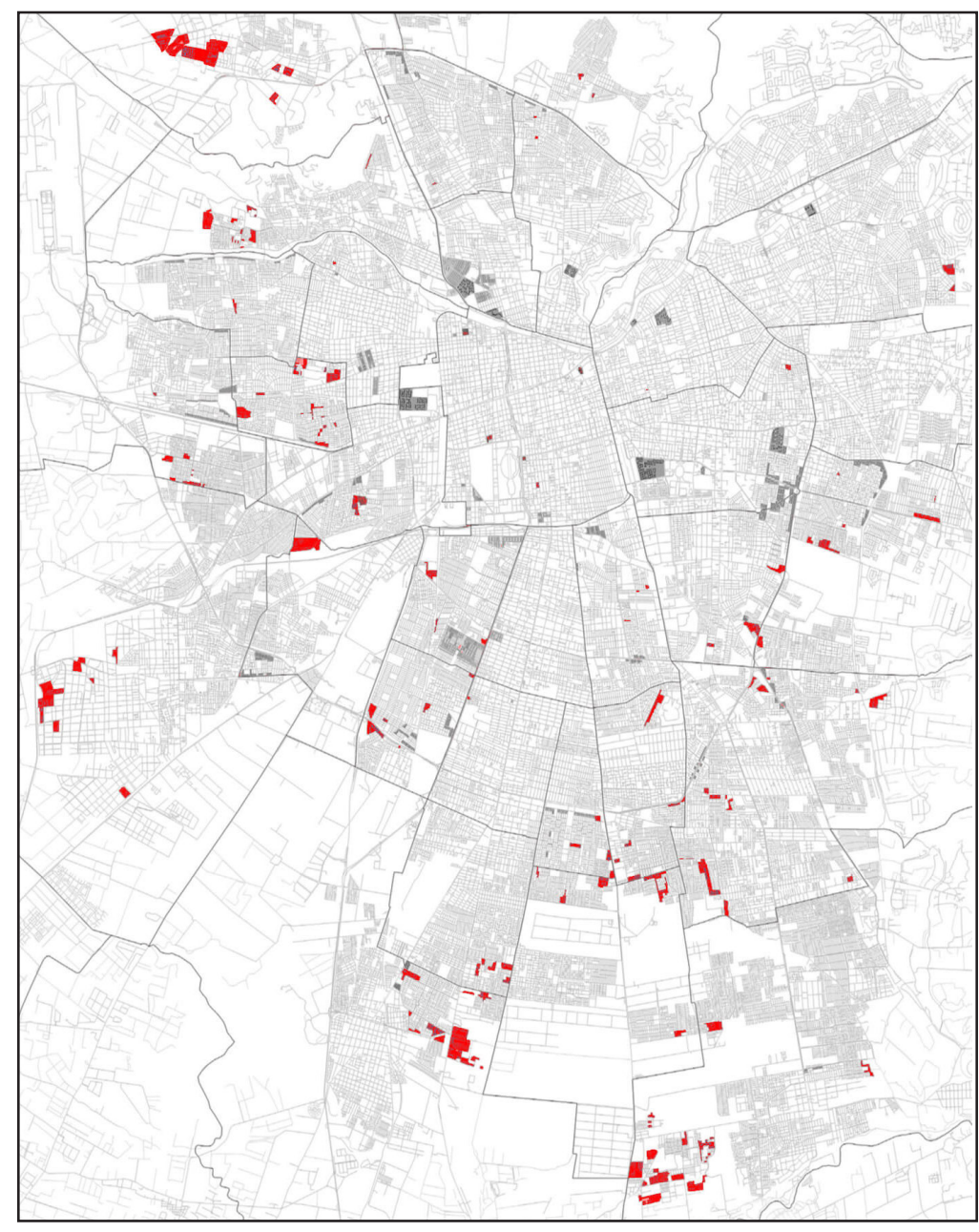


FIGURA 3. CONDOMINIOS SOCIALES EN ALTURA DE LA REGIÓN METROPOLITANA. CATASTRO REALIZADO POR CECILIA CAMARGO, BENJAMÍN CASTRO, SALVADOR ESCOBAR, FERNANDA EZEQUIEL, SIMÓN HERRERA, CHARLES LOUIS LAVANCHY, GIORGIO GANDINO, CONSUELO SAGAL Y VALENTINA SOTO.

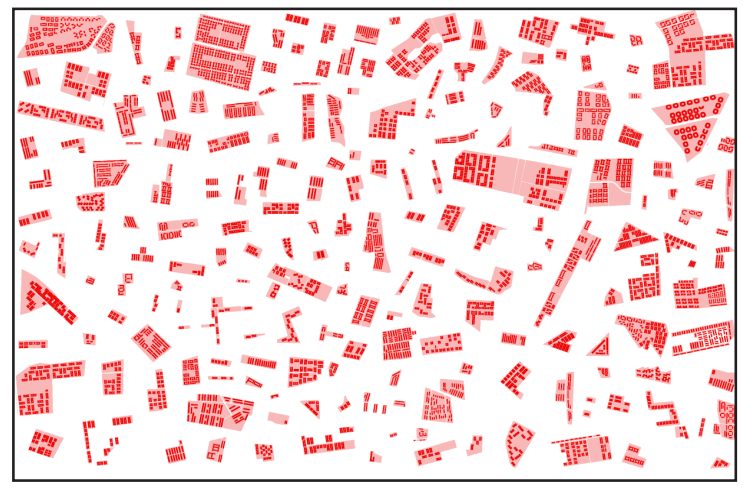

piso por parte de viviendas específicas, al cierre de cajas de escalera y pasillos o el enrejado de grupos de bloques o copropiedades, con el consiguiente abandono de grandes superficies que, al no contar con una definición clara en cuanto a su uso, propiedad y mantención promueven el encierro de los niños y adultos mayores dentro de las viviendas, para no exponerlos en espacios abiertos que se perciben como poco seguros (Salcedo, Hermansen y Rasse, 2017). Todo lo anterior ha llevado a la fragmentación de las comunidades vecinales, la extrema desconfianza entre vecinos, y que un grupo considerable de ellos desee abandonar su vivienda (Rodríguez y Sugranyes, 2005; Salcedo, Rasse y Cortés, 2013, Salcedo et al., 2017). Una encuesta aplicada en 2019 a 2.400 hogares residentes en 26 conjuntos de vivienda social en Santiago, Concepción y Talca muestra que el 50\% de los residentes preferiría irse del barrio si tuviera la posibilidad económica de hacerlo ${ }^{5}$.

En Chile existe un abanico amplio de políticas vinculadas a la vivienda colectiva en altura, cubriendo cuestiones básicas como la producción de nuevas unidades - orientada a subsanar el déficit cuantitativo existente en el país-a la rehabilitación ediliciay a regeneración barrial, centrada en las mejoras cualitativas de la vivienda y los entornos urbanos en que estas se desarrollan. Sin embargo, al momento de revisitar de forma específica la regeneración de condominios sociales en altura con bloques de tipo C, cobra especial importancia la oposición entre las operaciones de demolición y construcción de unidades de reemplazo y las acciones de regeneración y mejora de conjuntos habitacionales, actualizando la edificación y su entorno construido.

La primera representa una forma de intervención con un alto impacto mediático, cuyos efectos se registran fundamentalmente en el corto plazo, invisibilizando las externalidades negativas de la operación — pérdida de la energía invertida

$5 \quad$ FONDECYT 1171184 
FIGURA 4. CATASTRO, NÚMERO DE VIVIENDA Y DISTRIBUCIÓN TIPOLÓGICA DE BLOQUES C EN LA REGIÓN METROPOLITANA. CHARLES LOUIS LAVANCHY Y VALENTINA SOTO.

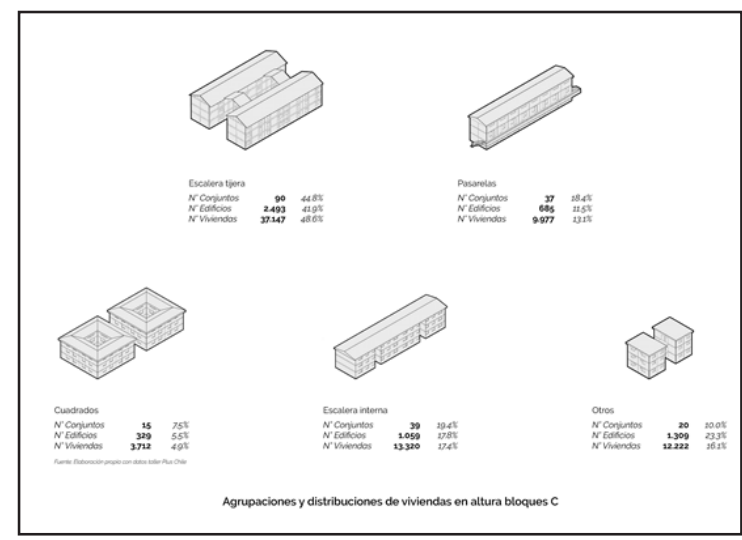

en la edificación, costos de demolición y reurbanización, destrucción del tejido social y desvalorización de la inversión estatal-, dejando gran parte de los problemas presentes en los condominios sociales en altura sin resolver; mientras que la segunda, caracterizada bajo el termino de regeneración, representa la oportunidad de transformar y reformar la ciudad, capitalizado la inversión que ya se ha realizado desde el Estado, mejorando y actualizando la vivienda y su entorno urbano, integrando cuestiones de orden tan diverso como pueden ser las deficiencias tecnológicoconstructivas de las edificaciones, la falta de equipamiento y áreas verdes, o la necesidad
FIGURA 5. CUADRO SE SUPERFICIE DE DEPARTAMENTO TIPO EN BLOCK C, DISCRIMINADO SUPERFICIE TOTAL Y SUPERFICIES ESPECIALIZADAS POR USO.

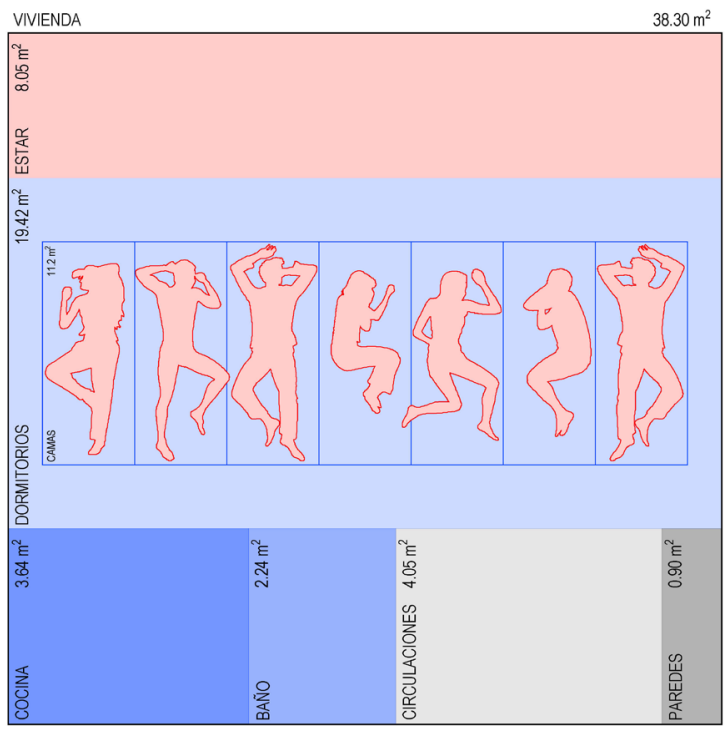

Fuente: Lavanchy, 2020.

de mejorar la conectividad con el transporte público de toda la ciudad.

Se estima que la mayoría de las viviendas colectivas en altura existentes permanecerán operativas al menos hasta el año 2050, por lo que las operaciones de regeneración y rehabilitación representan una posibilidad para extender el ciclo de vida de los edificios localizados en comunidades consolidadas. Esto es particularmente relevante en un contexto donde la construcción de vivienda nueva en las grandes áreas urbanas se ha vuelto cada vez 
FIGURA 6. LEVANTAMIENTO CON AMOBLADO DE DEPARTAMENTOS EN BLOCK C: A) DÚPLEX EN CONJUNTO BRASILIA, REGIÓN METROPOLITANA, B) DÚPLEX EN CONJUNTO BRASILIA, REGIÓN METROPOLITANA, C) DEPARTAMENTO EN PRIMERO PISO CON AMPLIACIÓN EN CONJUNTO BRASILIA, REGIÓN METROPOLITANA, D) DEPARTAMENTO EN CONJUNTO PADRES CARMELITOS. LAVANCHY, 2020.

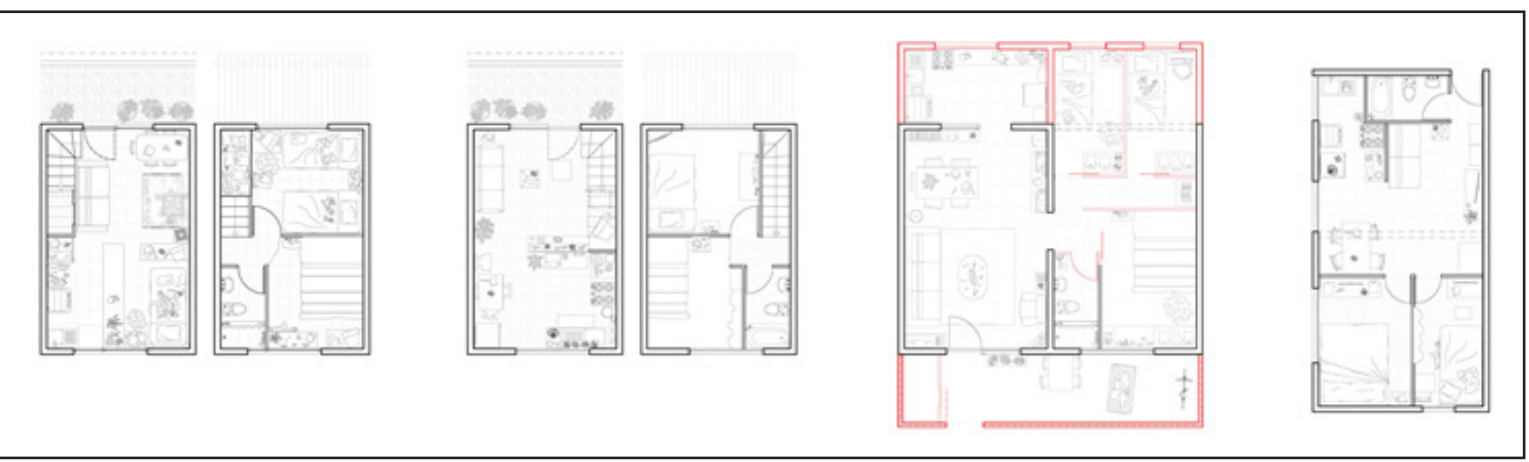

FIGURA 7. AMPLIACIONES IRREGULARES EN CONJUNTOS DE BLOCKS C. ELABORACIÓN PROPIA.
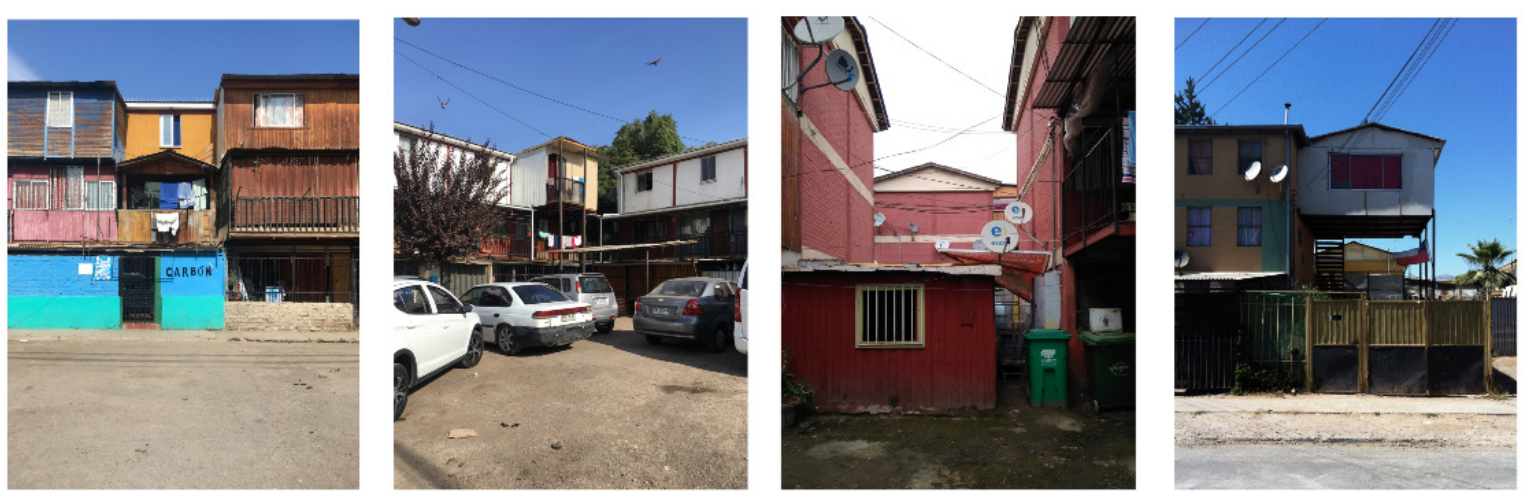
más complicada, producto del alza sostenida de los valores del suelo.

En el plano internacional, las estrategias de regeneración urbana y rehabilitación edilicia han cobrado relevancia, siendo particularmente significativo el desarrollo de casos en Europa Central — fundamentalmente en la rehabilitación y regeneración de vivienda construida luego de la segunda guerra mundial-, donde se suma a las operaciones promovidas desde el Estado el desarrollo de un marco teórico e investigación aplicada sobre el tema.

Los objetivos ambientales y energéticos de la Unión Europea para el año 2030 establecen una reducción de al menos $40 \%$ de gases de efecto invernadero y $32,5 \%$ de mejora de la eficiencia energética. El parque edificado representa el $40 \%$ del consumo de energía ("Assistance documents", 2013) y en 2012, 54 millones de europeos sufrían pobreza energética en sus viviendas, es decir, no era posible mantener una temperatura de confort interior ni solventar los gastos asociados (Tirado Herrero, Jiménez Meneses, López Fernández y Martín García, 2014). Es por esto que la intervención para mejorar el comportamiento energético de edificios existentes - y las condiciones de habitabilidad en general-se convirtió en un paso necesario para cumplir con la política europea (Cuchí y Sweatman, 2013).

Estudios sobre la rehabilitación de fachadas de edificios de vivienda colectiva en Suiza y Austria han demostrado una reducción significativa del consumo energético (80\%), de producción de CO2 (90\%) y una mejora de los índices de confort interior a lo largo del año (Miloni, Grischott y Zimmermann, 2011). Alternativas como ampliaciones por medio balcones y jardines de invierno han sido implementadas en edificios en altura en Francia por Druot, Lacaton y Vassal (2007), mientras que su impacto en la disminución del consumo de energía ha sido estudiado mediante casos construidos y simulaciones en distintos climas en España (Monge-Barrio y Sánchez-Ostiz, 2015) e Italia (Fotopoulou, Semprini, Cattani, Schihin, Weyer, Gulli y Ferrante., 2018).

El alcance de los proyectos de rehabilitación no sólo se enmarca en el ámbito tecnológico, sino que también considera dimensiones en los aspectos económico y social. Se ha determinado que la factibilidad de distintas estrategias de rehabilitación debe considerar las características del edificio y depende no sólo de la tecnología disponible, sino de restricciones económicas y legales (Dall'O, Galante y Pasetti, 2012). La rentabilidad de los proyectos de rehabilitación con mejoramiento de envolvente e incorporación de espacios intermedios ha sido estimada con un período de retorno de 10 años (Ferrante y Semprini, 2011). Mientras, en las propuestas que consideran la densificación de los conjuntos se ha determinado que la alta inversión inicial puede ser compensada por medio de la venta de las nuevas unidades (Paiho, Ketomaki, Kannari, Hakkinen y 
Shameikka, 2019). Se estima que, en Europa, la rehabilitación de 10 millones de viviendas hasta 2050 podría crear más de 150.000 empleos, un mercado de entre 2.000 y 10.000 millones de euros anuales y una revalorización de la vivienda de hasta un 10\% (Cuchí y Sweatman, 2013).

Desde el punto de vista social, se han estudiado las conductas de los usuarios y su impacto en el consumo de energía del edificio (D’Oca y Veld, 2018). La rehabilitación de edificios en las ciudades representa el mayor desafío en términos de reducción de emisiones $\mathrm{CO} 2$, pero también produce el mayor impacto en reactivar barrios existentes al afectar positivamente el entorno construido (Ferrante y Semprini, 2011). A partir de las experiencias desarrolladas en España, el 76\% de los municipios constatan efectos de revitalización social y económica como uno de sus resultados (Casanovas, Cuchí, Mas Herrero y Rubio del Val, 2018). Estudios en Croacia destacan que la mejora de calidad de vida de los beneficiarios reduce el gasto en programas sociales y que el déficit público es neutro aun a corto plazo (Mikulic, Rasic Bakaric y Slijepcevic, 2016).

Estos casos a nivel internacional avalan la rehabilitación de la vivienda colectiva como una alternativa social, energética y económicamente sustentable para extender el ciclo de vida de los edificios. Sin embargo, hoy solo el 1,2\% del parque de vivienda colectiva en altura es rehabilitado al año ("Assistance documents", 2013) y la actividad en este sector presenta un crecimiento menor a lo esperado. Según Casanovas et al. (2018) algunas de las limitaciones del modelo aplicado a ese tipo de programas se relacionan con: a) la falta de un diagnóstico que identifique la diversidad de situaciones existentes y promueva la rehabilitación; b) la inapropiada asignación de los subsidios o fuentes de financiamiento para las comunidades y c) falencias en los plazos de las convocatorias, procesos de gestión y coordinación de recursos.

Los estudios realizados han identificado el impacto positivo que pueden tener estas intervenciones en distintos ámbitos, sin embargo, la correcta implementación de los programas de regeneración urbana y de políticas públicas es clave para facilitar la gestión de estas propuestas.

\section{El Programa de Regeneración de Conjuntos Habitacionales y sus proyectos}

\begin{abstract}
El Ministerio de Vivienda y Urbanismo crea en el año 2017 el Programa de Regeneración de Conjuntos Habitacionales (PRCH) que establece la necesidad urgente por reducir el déficit cualitativo de la vivienda social y mejorar los edificios de condominios en altura. El objetivo
\end{abstract}


principal del programa es regenerar ciertos conjuntos habitacionales de viviendas sociales altamente deteriorados y sus entornos (Decreto 18, 2018). La mejora de la calidad de vida de las familias que habitan estos conjuntos deteriorados se realiza mediante un enfoque interdisciplinario basado en estudios sociales, jurídicos, habitacionales y urbanos. Metodológicamente, los estudios se traducen en un plan maestro que utiliza un diseño participativo de acuerdo a las necesidades particulares de cada conjunto (MINVU) y entre los objetivos específicos que el programa considera está la rehabilitación o reconstrucción de viviendas, la reconfiguración del espacio público de los conjuntos y la gestión de la movilidad habitacional de las familias, apoyándose en un trabajo de organización comunitaria.

De acuerdo a Villagra (2018), entre 2000 y 2018, el MINVU ha desarrollado 18 casos de intervención en condominios sociales en altura que se pueden clasificar en tres grupos: a) acciones sin enmarcar en ningún programa de intervención concreto (SPI), realizadas en un período en que el MINVU carecía de instrumentos específicos orientados a la regeneración de conjuntos de vivienda; b) intervenciones realizadas bajo el Programa "Segunda oportunidad" (2da), cuyo objetivo es demoler bloques deteriorados y otorgar subsidios a sus residentes, para que busquen individualmente una nueva vivienda; y c) intervenciones realizadas bajo el Programa de Regeneración de Conjuntos Habitacionales $(\mathrm{RCH})$. Aun cuando el alcance de dichos programas es similar en número de viviendas, los proyectos desarrollados por el Programa de Regeneración de Conjuntos Habitacionales, al rehabilitar las viviendas existentes, incorporan un porcentaje de demolición considerablemente menor a los programas predecesores.

En un principio, las intervenciones de rehabilitación eran casos aislados que consideraban un aprendizaje sobre la marcha, donde no existía una sistematización del proceso requerido. La gestión de los primeros proyectos ha sido realizada por medio de la combinación de diversos instrumentos y partidas presupuestarias, tanto desde el MINVU como de otros ministerios. Sin embargo, todas estas intervenciones presentan problemáticas y variables críticas similares situaciones legales, estructuras morfológicas y problemáticas socioeconómicas semejantes-, posibilitando la elaboración de un modelo de gestión de proyectos para la regeneración de condominios sociales en altura desde el MINVU (la existencia de este modelo es posterior a la implementación de los primeros casos de intervención).

El modelo de gestión declarado por el programa de regeneración de conjuntos contempla tres fases, organizadas de acuerdo con lo expresado en el Decreto 18 (2018): a) diagnóstico, b) elaboración del plan maestro y c) ejecución y cierre. Cada fase se compone de actividades, hitos y productos 
TABLA 1: CASOS DE INTERVENCIÓN EN CONDOMINIOS SOCIALES DESARROLLADOS ENTRE 2000 Y 2018.

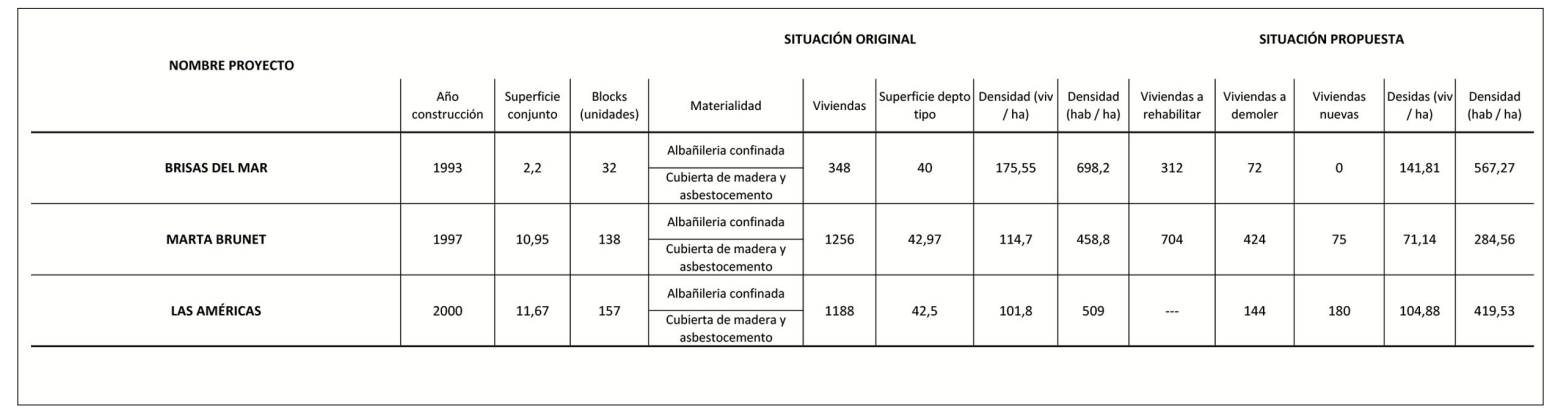

vinculados con las diferentes dimensiones que se abordan a lo largo del proceso.

Fase 1 - Diagnóstico: considera un plazo de aplicación de 11 meses. Un equipo del gobierno coordina la conformación del comité de regeneración y la ejecución de diagnóstico en las áreas habitacional, urbana, social y jurídica. A partir de los resultados del análisis se procede a conformar una mesa técnica con el objetivo de definir las directrices del plan maestro. Dicha instancia está conformada por el director del SERVIU correspondiente, el Secretario Regional del MINVU, y el jefe de la División de Desarrollo Urbano.

Fase 2 - Elaboración del plan maestro: integra la información derivada de la etapa anterior y se desarrollan planes de
Gestión Habitacional, Urbana y Social, considerando el diseño participativo con la comunidad y la SEREMI correspondiente. El período estimado para esta fase es de 8 meses de duración.

Fase 3 - Ejecución y cierre: Considera todas las acciones y obras definidas en la etapa del plan maestro y es monitoreada por medio de informes de avance anuales. Al entregar a la comunidad las obras finalizadas se procede con la etapa de cierre y traspaso de la gestión del conjunto rehabilitado. Esta fase es la que considera un mayor período de ejecución, estimado en 60 meses. Sin embargo, hasta el año 2019, ninguna intervención en desarrollo ha implementado el cierre del proyecto, dando por finalizado el proceso contemplado por el Programa 
de Regeneración de Conjuntos Habitacionales.

Dentro de las variables involucradas en el proceso, la extensión en el tiempo pareciera ser una de las más gravitantes, ya que afecta directamente la participación de e las comunidades involucradas, encarece los costos asociados a la operación e incrementa el nivel de incertidumbre al extenderse más allá del ciclo de gobernanza de las autoridades involucradas en su gestión y ejecución. Desde el MINVU se estima que el proceso completo debiera demorar aproximadamente 7 años (Villagra, 2018). Sin embargo, las intervenciones en desarrollo han involucrado un largo proceso de aprendizaje donde la falta de sistematización y la aparición de requerimientos específicos a cada caso han dificultado su ejecución. La gestión ha requerido combinar diversas políticas públicas, ya que no existe un subsidio específico dirigido a la rehabilitación de condominios sociales en altura, debiendo utilizarse herramientas de financiamiento que pueden (o no) ajustarse a los distintos requerimientos que implica cada plan de rehabilitación. La experiencia ha evidenciado los importantes impactos sociales y económicos que tienen los largos periodos de ejecución en la comunidad, convirtiéndose la reducción o compresión de los plazos en una preocupación central al momento de planificar operaciones de regeneración. Los proyectos de regeneración deben tener en cuenta la incertidumbre que puede suscitar entre comunidades expuesta situaciones de confrontación y minimizar los aspectos difíciles de afrontar por los vecinos. En la actualidad, las experiencias en desarrollo permiten identificar patrones replicables que constituyen nudos críticos en los actuales programas de rehabilitación. Estos hitos pueden extender los tiempos de ejecución de los proyectos, comprometiendo así su factibilidad social y económica y arriesgando la permanencia de este tipo de programas en la oferta pública futura.

\section{Analizar los procesos PRCH para mejorarlos}

A partir de la identificación y análisis de los hitos críticos de los proyectos de regeneración y rehabilitación en condominios sociales en altura en Chile, es posible identificar las variables críticas involucradas en el proceso y establecer diferentes jerarquías entre ellas, diferenciado inicialmente entre "dimensiones esenciales" y "dimensiones complementarias", de acuerdo al rol que tiene en las tres fases del proceso (diagnóstico, elaboración del plan maestro y ejecución y cierre).

La investigación se centra en el estudio de tres casos - Brisas del Mar-Nuevo Horizonte, Marta Brunet y Las Américas-, correspondientes a conjuntos habitacionales intervenidos por el programa de regeneración de conjuntos habitacionales del MINVU. 
Se desarrolló un análisis de casos considerando su gestión e implementación para reconstruir la historia de cada uno de ellos, las decisiones tomadas, sus justificaciones, las principales dificultades, y los resultados. Metodológicamente, se pueden mencionar tres ámbitos de acción: a) revisión de documentos e informes relativos al programa de regeneración de conjuntos habitacionales del MINVU, b) entrevistas a informantes clave del proceso y c) visita y observación de los casos de conjuntos intervenidos para generar una visión integral del proceso. La información fue sistematizada en una matriz de análisis, organizada de acuerdo con fases de aplicación del proyecto, distinguiendo entre actores, recursos y procesos en cada etapa. Con esto, se identificaron variables o dimensiones comunes en el desarrollo de los procesos y sus hitos críticos.

A partir de los resultados, se elaboraron recomendaciones, orientadas a mejorar el proceso de gestión y aplicación, que permitan reducir plazos, disminuir costos, eliminar procesos redundantes y su posterior evaluación en escenarios futuros de propuestas de intervención.

\section{Tres casos de estudio PRCH}

Dentro de los 18 casos de intervención desarrollados por el MINVU hasta 2018, se seleccionaron tres casos representativos: los conjuntos Brisas del Mar-Nuevo Horizonte II (BM) localizado en Viña del Mar, Región de Valparaíso; Marta Brunet (MB) en Santiago, Región Metropolitana; y Las Américas (LA) en Talca, Región del Maule (Tabla 2), pues, en conjunto, permiten caracterizar las diferentes problemáticas vinculadas a la rehabilitación y regeneración de condominios sociales en altura en Chile.

\section{BRISAS DEL MAR}

El conjunto Brisas del Mar representa uno de los casos emblemáticos de rehabilitación con ampliación de departamentos en Chile. Las viviendas fueron construidas en 1993 como parte del Programa de Vivienda Básica en un complejo terreno con pendiente en la periferia de la ciudad de Viña del Mar. Este conjunto presentaba problemas graves de hacinamiento, sistema de alumbrado público precario y cierros entre los bloques y microbasurales que propiciaban la presencia de plagas. La reducida 
FIGURA 8. BRISAS DEL MAR, NUEVO HORIZONTE, MARTA BRUNET Y LAS AMÉRICAS.
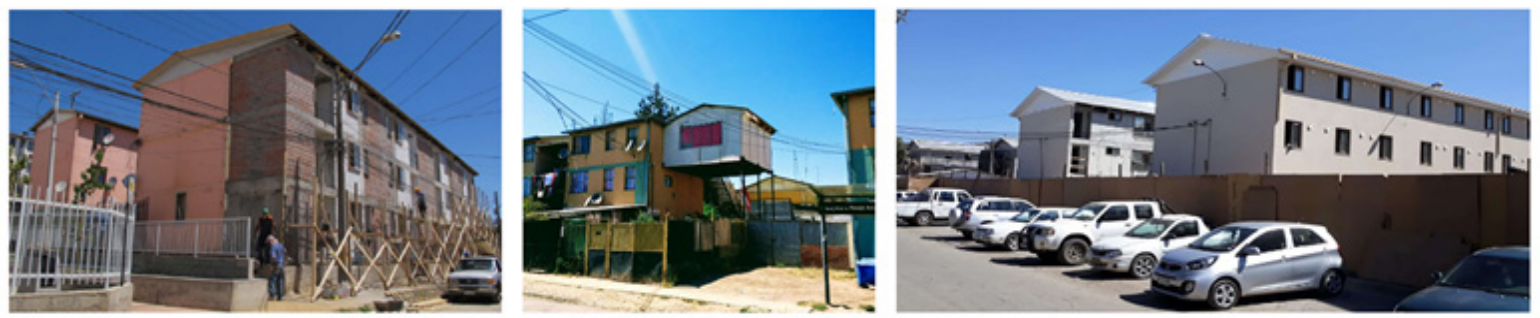

Fuente: Elaboración Propia

FIGURA 9. CASOS DE ESTUDIOS: BRISAS DEL MAR, NUEVO HORIZONTE, MARTA BRUNET Y LAS AMÉRICAS.

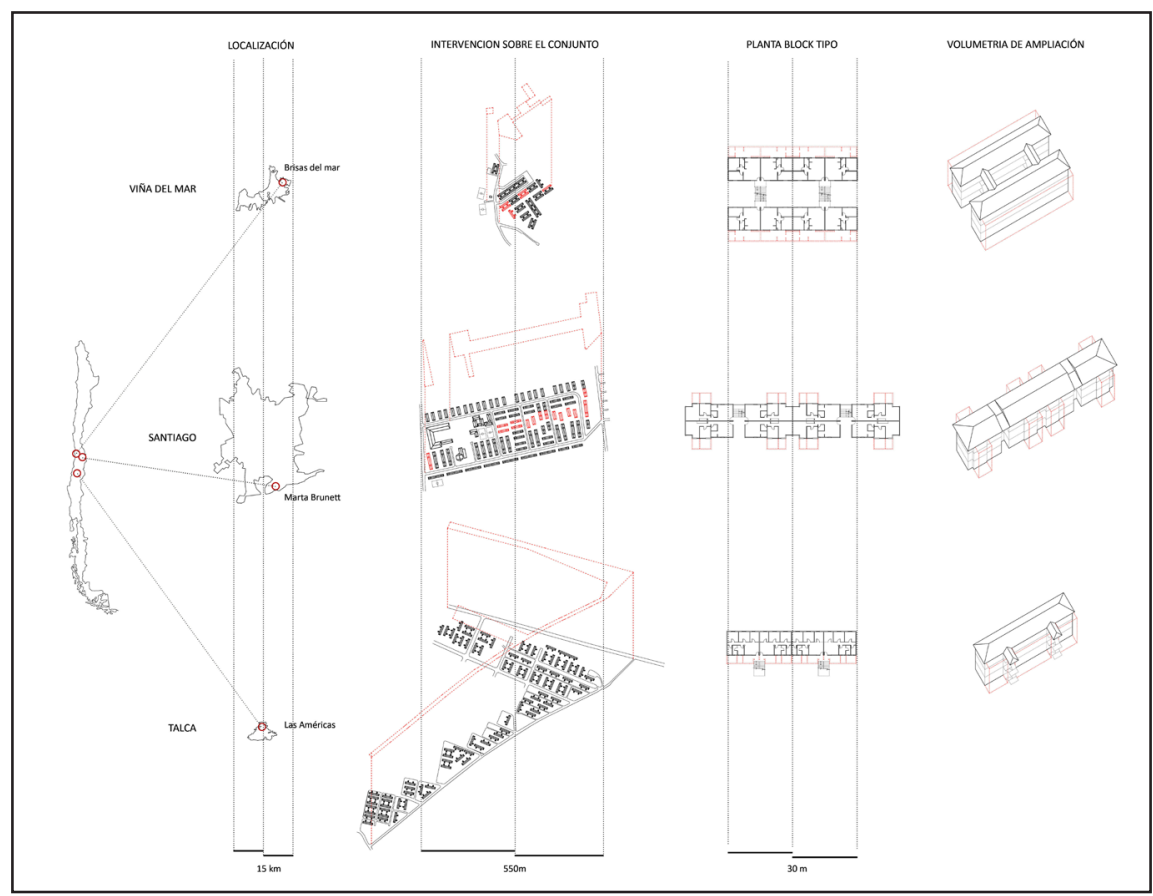

Autor: Francisco Chateau 
superficie de los departamentos llevó a los vecinos a tomarse terrenos de forma ilegal.

El año 2012 se inicia la implementación del Programa Segunda Oportunidad por parte del MINVU. Este programa consideró la demolición de un $18 \%$ de los edificios existentes con el objetivo de proveer espacio para el uso público. A partir de los resultados del diagnóstico realizado en simultáneo y el trabajo con los vecinos, surge la propuesta de mantener a una parte de los habitantes en el lugar y de rehabilitar sus viviendas. Los departamentos rehabilitados mejoran su envolvente térmica e incrementan su superficie de 40 a $57 \mathrm{~m} 2$. Las ampliaciones son diseñadas como un nuevo edificio de $2,5 \mathrm{~m}$. de espesor estructurado en albañilería con un comportamiento estructural independiente. En 2017 se inicia la construcción de la ampliación que posee la particularidad de ser implementada con las familias habitando sus departamentos. Para las obras se estimó una duración original de 11 meses, sin embargo, la primera etapa se llevó a cabo en 18 meses y la intervención presentaba un nivel de avance estimado del 26\% sobre el total de departamentos a rehabilitar para el año 2019, mientras que la totalidad de los habitantes que dejaron sus viviendas como parte del Programa Segunda Oportunidad ya habían sido reinstalados en nuevas viviendas.

\section{Marta Brunet}

El conjunto Marta Brunet, se localiza en la comuna de Puente Alto —en la zona conocida como Bajos de Mena- en la Región Metropolitana. Luego de su construcción, el conjunto presentó problemas con el temporal de lluvia de 1997 — el caso de las denominadas casas "COPEVA" corresponde al mismo episodio y se encuentra en la misma zona de la comuna-, lo que lo caracterizó como uno de los conjuntos con mayor deterioro en la zona. A la deficiente calidad constructiva de sus viviendas se suma un alto número de ampliaciones informales que repercuten en una disminución y deterioro de los espacios de uso público.

El año 2015, el conjunto fue seleccionado para ser intervenido por el programa de $\mathrm{RCH}$ en conjunto con el Plan Integral de Bajos de Mena. Entre julio 2016 y enero 2017 se desarrolló un plan maestro que consideraba disminuir el número de viviendas a 1.256 , programando la rehabilitación de 779 unidades y la demolición de las viviendas restantes con el objetivo de liberar suelo y construir un parque en el eje central del conjunto. Las familias que dejan sus viviendas son beneficiadas con un programa de movilidad que les otorga la opción participar de un Subsidio de Construcción de Vivienda 
TABLA 2. SITUACIÓN ORIGINAL Y PROPUESTA DE LOS CASOS DE ESTUDIO.

\begin{tabular}{|c|c|c|c|c|c|c|c|c|c|}
\hline & & & & & & & \multicolumn{3}{|c|}{ Programa y año de implementación } \\
\hline & $\begin{array}{l}\text { Región de interven- } \\
\text { ción }\end{array}$ & Nombre proyecto & $\begin{array}{l}\mathrm{N}^{\circ} \text { vivien- } \\
\text { das }\end{array}$ & \multicolumn{3}{|c|}{$\begin{array}{l}\text { Superficie } \\
\text { vivienda } \mathrm{m}^{2}\end{array}$} & $\begin{array}{c}\text { Spl } \\
2000-2012\end{array}$ & $\begin{array}{c}2 \mathrm{da} . \\
2012-2015\end{array}$ & $\begin{array}{c}\text { Rch } \\
2017 \text { - presente }\end{array}$ \\
\hline & Tarapacá & Las Quintas I, II y II & 552 & \multicolumn{3}{|c|}{39} & & & \\
\hline & Metropolitana & Las Viñitas & 1.029 & \multicolumn{3}{|c|}{36} & & & \\
\hline & Metropolitana & Villa San José II & 1.708 & \multicolumn{3}{|c|}{42,5} & & & \\
\hline & Bio Bio & Valle San Pedro & 600 & \multicolumn{3}{|c|}{50} & & & \\
\hline & Bio Bio & Villa Futuro & 1.320 & \multicolumn{3}{|c|}{50} & & & \\
\hline & Araucanía & Villa los Cóndores & 900 & \multicolumn{3}{|c|}{42} & & & \\
\hline \multirow{7}{*}{$\begin{array}{c}\text { Caso de } \\
\text { estudio } \\
1\end{array}$} & Valparaíso & $\begin{array}{l}\text { Brisas del Mar - Nuevo } \\
\text { Horizonte II }\end{array}$ & 348 & \multicolumn{3}{|c|}{40} & & & \\
\hline & Metropolitana & Parinacota I y II & 1.793 & 40 & 43 & 51 & & & \\
\hline & Metropolitana & $\begin{array}{l}\text { Francisco Coloane-Cerro } \\
\text { Morado }\end{array}$ & 1.356 & $\begin{array}{l}42 \\
25\end{array}$ & & $\begin{array}{l}43 \\
98\end{array}$ & & & \\
\hline & $\begin{array}{l}\text { General Libertador } \\
\text { Bernardo O'Higgins }\end{array}$ & $\begin{array}{l}\text { Vicuña Mackenna I, II } \\
\text { y III }\end{array}$ & 1.793 & 40 & 43 & 51 & & & \\
\hline & Antofagasta & Villa Jorge Alessandri & 256 & & 32,25 & & & & \\
\hline & Coquimbo & Población Baquedano & 118 & Vivie & enda ais & lada & & & \\
\hline & Valparaíso & San Agustín & 224 & & 47 & & & & \\
\hline \multirow{4}{*}{$\begin{array}{l}\text { Caso de } \\
\text { estudio } \\
2\end{array}$} & Metropolitana & Marta Brunet & 1.256 & \multicolumn{3}{|c|}{42,97} & & & \\
\hline & Metropolitana & Santa Julia & 2.111 & \multicolumn{3}{|c|}{ Vivienda aislada } & & & \\
\hline & Metropolitana & Nacimiento & 357 & \multicolumn{3}{|c|}{ Vivienda aislada } & & & \\
\hline & $\begin{array}{l}\text { General Libertador } \\
\text { Bernardo O'Higgins }\end{array}$ & Baltazar Castro & 660 & \multicolumn{3}{|c|}{40} & & & \\
\hline \multirow{6}{*}{$\begin{array}{l}\text { Caso de } \\
\text { estudio } \\
3\end{array}$} & Maule & $\begin{array}{l}\text { Las Américas II, V, VI, } \\
\text { VII, VIII y X }\end{array}$ & 1.188 & \multicolumn{3}{|c|}{42,5} & & & \\
\hline & De los Ríos & San Pedro & 256 & 42,6 & & 54 & & & \\
\hline & $\begin{array}{l}\text { Total proyectos por } \\
\text { programa }\end{array}$ & & & & & & 6 & 4 & 9 \\
\hline & $\begin{array}{l}\text { Total viviendas por } \\
\text { programa }\end{array}$ & & & & & & 6.109 & 5.213 & 6.426 \\
\hline & $\begin{array}{l}\text { Habitantes x programa } \\
\text { (aproximados) }\end{array}$ & & & & & & 23.235 & 20.640 & 26.865 \\
\hline & $\begin{array}{l}\text { Porcentaje de demoli- } \\
\text { ción x programa }\end{array}$ & & & & & & $100 \%$ & $90 \%$ & $41 \%$ \\
\hline
\end{tabular}

Fuente: elaboración propia.

Chateau, F., Schmitt, C., Rasse, A., y Martínez, P. (2019). Consideraciones para programar la regeneración de condominios sociales en

revista invi 159 altura. Estudio comparado de tres casos en Chile. REVISTA INVI, 35 (100), 143-173 doi:10.4067/S0718-83582020000300143 
FIGURA 10. AXONOMÉTRICA, CON INTERVENCIÓN SOBRE BLOCK C EN BRISAS DEL MAR. SE REPRESENTA LA AMPLIACIÓN, LA INTERVENCIÓN EN LAS INSTALACIONES SANITARIAS Y EL CAMBIO DE LA CUBIERTA. SOTO, 2020.

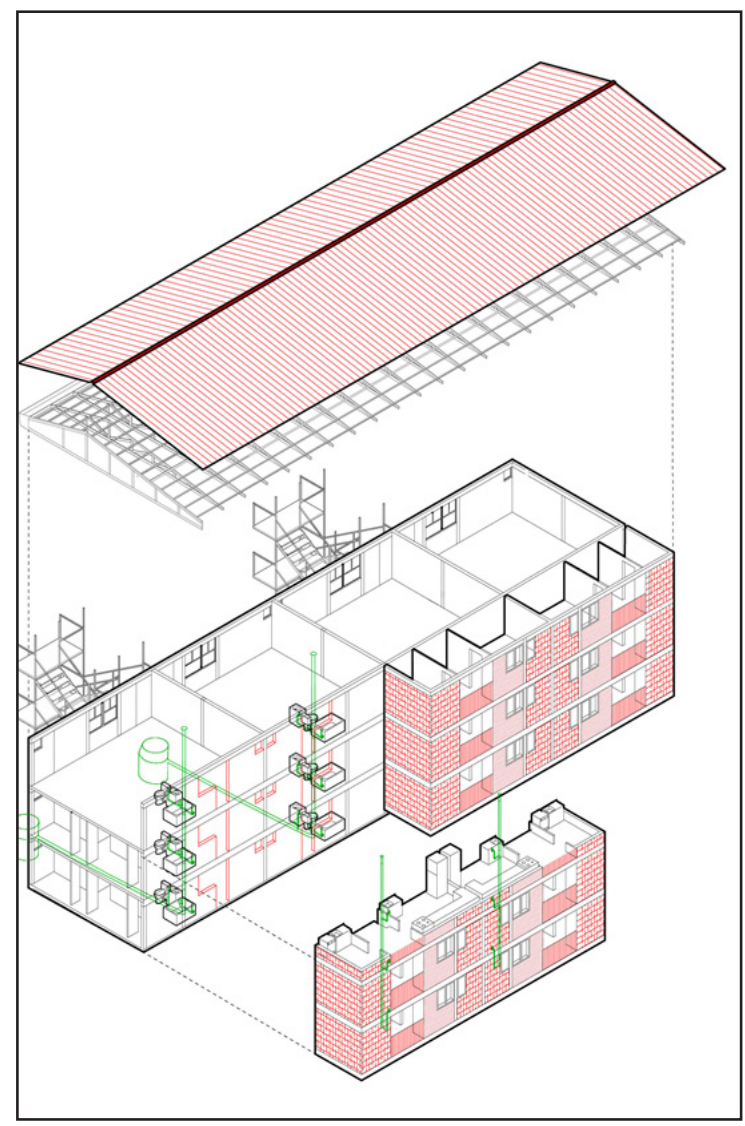

en Nuevos Terrenos (CNT) dentro de la misma comuna o la compra de la vivienda por parte del Estado para buscar otras alternativas.

A mediados de 2018 el plan maestro es sometido a modificaciones para adaptarse a las nuevas preferencias de los vecinos relacionadas con su alternativa de solución habitacional. En septiembre de dicho año se da inicio a la demolición de los primeros bloques y se estima que las obras se completarán para 2025. En el año 2019 el proyecto presenta aproximadamente un $15 \%$ de avance.

\section{Las Américas}

El conjunto Las Américas fue construido el año 2000 en la periferia norte de Talca, presentando una alta densidad urbana, situándolo como uno de los sectores con mayor concentración de habitantes de la Región donde es posible constatar un alto deterioro de los espacios comunes y los departamentos. En enero de 2015 el Programa de Regeneración de Conjuntos Habitacionales decide intervenir el conjunto Las Américas, conformando una mesa de trabajo territorial, compuesta por el equipo del programa "Quiero mi barrio" y un equipo del MINVU, que cuenta con una contraparte vecinal, consistente una mesa territorial de dirigentes del sector, con apoyo de ONGs locales. En este marco, se desarrolla el diagnóstico del conjunto. 
FIGURA 11. EVOLUCIÓN CRONOLÓGICA DE LA INTERVENCIÓN EN BRISAS DEL MAR.
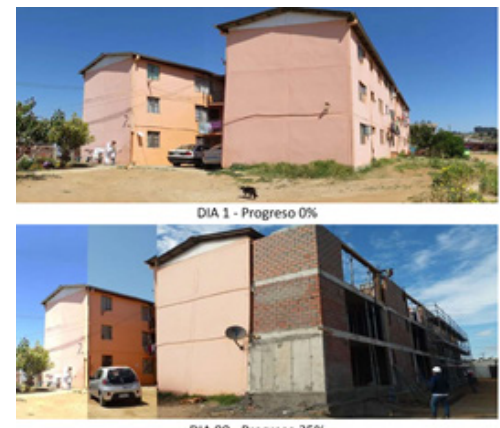

DA 90 - Progreso $35 \%$
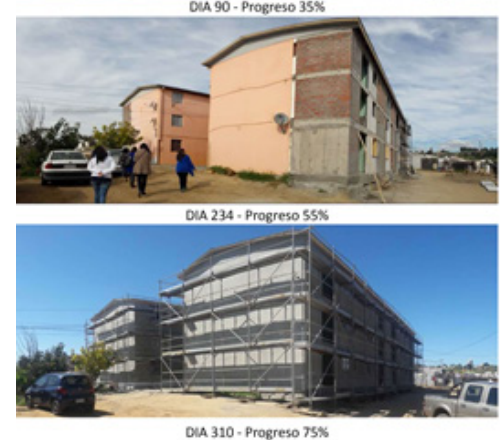

Fuente: SEREMI MINV
J. trabajadores sociales.

Se solicita un estudio estructural financiado por el PNUD y elaborado por el IDIEM que determina que es viable la rehabilitación de las estructuras edilicias existentes. En forma simultánea se elabora un diagnóstico legal que identifica una situación de alta complejidad. Aproximadamente un $70 \%$ de los habitantes son propietarios, mientras que el porcentaje restante se compone de arrendatarios y departamentos ocupados ilegalmente y localizados en prácticamente todos los bloques. Además, se realiza un censo poblacional para caracterizar el perfil de los habitantes, sus intenciones de permanecer en el conjunto y sus expectativas respecto al programa de intervención. 
En el año 2016 se toma la decisión de no demoler las viviendas, para emprender el proceso regeneración y la elaboración de un plan maestro. El diseño del plan maestro de intervención considera la demolición de sólo 144 viviendas, para construir un centro cívico barrial destinado a convertirse en un espacio de integración social, a través del mejoramiento de los equipamientos, la iluminación y las áreas verdes. En términos habitacionales, se propone un diseño de 5 etapas para rehabilitar las viviendas restantes, mejorar sus instalaciones y ampliar su superficie. Durante este período los vecinos serán parte de un plan de movilidad habitacional temporal para permitir las obras en los departamentos.

En 2018 se inician las obras de la primera etapa de un total de 1.188 departamentos, involucrando aproximadamente a 5.000 habitantes. A principios de 2019, el estado de avance actual del proyecto se estima en un $10 \%$.

\section{Una cronología ideal para un proceso real}

El análisis se estructura en dos segmentos. El primero se centra en un cronograma comparativo de los casos de estudio con los plazos estimados y recomendados por el Programa de Regeneración de Condominios Habitacionales del MINVU. La revisión de este cronograma permite comprender la lógica detrás de la planificación de las actividades y la concordancia de los plazos establecidos respecto de la experiencia efectiva en cada conjunto. En un segundo segmento, se trabaja sobre las etapas del proyecto, identificando las dimensiones comunes de cada intervención, relevando aquellas que tienen mayor importancia para la implementación, afectando los plazos de ejecución. Estas dimensiones se dividen en dos grupos: 1) "dimensiones esenciales", que se clasifican como imprescindibles para el desarrollo del proyecto de regeneración (de acuerdo con el relato de los informantes), y 2) "dimensiones complementarias" que representan actividades adicionales, cuya ejecución refuerza el proceso o soluciona aspectos secundarios. Cada una de estas dimensiones considera los agentes que participan en su ejecución y/o implementación, una fase de aplicación y una duración que afectan directamente la ejecución de las intervenciones. Se desarrolla un análisis para cada una de estas dimensiones.

\section{Cronogramas}

El cronograma considera el avance de los casos de estudio hasta inicios de 2019. Los tres casos estudiados aún no concluyen la fase de implementación de los proyectos por lo que no ha sido posible analizar las actividades de evaluación, seguimiento con la comunidad y de cierre. Para la etapa de construcción se 
considera una complementación entre el grado de avance las obras de regeneración (entre 10\% y $26 \%$ ) y los plazos estimados para los trabajos proyectados según los equipos de $\mathrm{RCH}$.

Según esta información, es posible observar las siguientes características:

a) Los tres casos de estudio se encuentran abiertos y con distintos niveles de avance, por lo que los plazos totales de implementación de los proyectos no se pueden referenciar con exactitud. La fase de ejecución presenta un alto grado de incertidumbre debido a los muchos imprevistos en obra que todavía podrían afectar los plazos estimados. Hasta el año 2019 todas las obras presentaban un retraso de al menos 6 meses respecto a lo contemplado en los plazos originales.

b) Los casos de estudio presentan tiempos de ejecución que superan los períodos recomendados por el MINVU. Por una parte, la fase de inserción y diagnóstico de los casos estudiados fueron desarrolladas antes de la elaboración de las fases definidas por el RCH. Sin embargo, aún es posible identificar de qué manera las dimensiones esenciales podrían reducir los tiempos de ejecución.

c) El proceso de implementación sugerido por el MINVU consiste en una aplicación lineal de las distintas fases. Sin embargo, los casos estudiados muestran una superposición de las fases en un proceso no-lineal. Esta condición permitiría evaluar de forma simultánea la implementación de determinadas dimensiones para hacer más eficiente la aplicación de la intervención.

d) La fase de elaboración del plan maestro presenta períodos similares a los recomendados por el MINVU en los casos de Marta Brunet y Las Américas. Sin embargo, el plan maestro puede ser sujeto a rediseño y a evaluaciones necesarias sobrepuestas a la fase de ejecución, como ocurre en Marta Brunet. Estas consideraciones deben ser previstas en la elaboración de un modelo de gestión con una implementación más flexible.

\section{Dimensiones}

A partir de los casos estudiados se identificaron patrones en el desarrollo de las propuestas relevantes en su implementación y condicionantes de los plazos de ejecución, calificando las variables o dimensiones involucradas en dos grupos (esenciales y complementarias).

\section{Diagnóstico}

El diagnóstico legal y el diagnóstico estructural (aun cuando este último no es considerado explícitamente por las recomendaciones del MINVU) resultan imprescindibles para 
determinar la factibilidad de ampliar o remodelar los edificios existentes. Ambos diagnósticos son identificados como dimensiones esenciales por lo que deberían ser implementados al comienzo de la intervención, reduciendo la incertidumbre sobre el total de la operación. Los casos de estudio han complementado estos diagnósticos con encuestas a los residentes y análisis del desempeño térmico y del estado de las instalaciones en las viviendas.

El diagnóstico social (identificación de las organizaciones del barrio, mapeo de actores y redes, recursos del territorio, casos críticos) y el involucramiento de los vecinos desde las primeras etapas del proyecto resulta central. Dado que el proyecto es invasivo y tiene etapas que necesitan de una alta colaboración entre la comunidad y el equipo del proyecto (desde el diseño del plan maestro, hasta la coordinación de la movilidad residencial), se requiere una relación de largo plazo y de confianza. Asimismo, es necesario evitar la propagación de rumores que entorpezcan el trabajo conjunto y desarrollar estrategias para manejar adecuadamente las expectativas de los residentes frente a un proyecto que, al ser tan abierto e involucrar aspectos muy sensibles (el patrimonio de las familias materializado en las viviendas, el lugar de residencia durante la movilidad residencial, entre otros), deja muchos espacios de incertidumbre.
En este marco, el temprano trabajo comunitario del equipo de proyecto, la sociabilización con los dirigentes y vecinos, la declaración de los objetivos, la explicación oportuna y clara de las posibilidades y restricciones tanto del proyecto como del marco temporal en que se desarrollarán las diferentes etapas que éste involucra -qué tipo de cambios pueden incorporarse y cuáles no, la incorporación (o no) de movilidad, entre otros- resulta fundamental. Asimismo, los dirigentes deben tener acceso directo a los profesionales a cargo de la intervención, de forma de poder consultar y aclarar las dudas que les manifiesten los vecinos, manteniendo a la comunidad alineada con el proyecto, tratando de reducir al máximo la propagación de información errónea.

\section{Movilidad de los residentes durante la construcción}

La mayoría de los proyectos de regeneración consideran el traslado temporal de sus habitantes para poder realizar las obras de construcción -la intervención sobre las instalaciones sanitarias representa una de las partidas críticas en este sentido-, sumando un costo indirecto a la operación de rehabilitación, aumentando la reticencia por parte de los vecinos debido a las dificultades cotidianas que esto les genera (aumento de distancia a la escuela o al 
TABLA 3. CRONOGRAMA COMPARATIVO FASES MINVU, BRISAS DEL MAR, MARTA BRUNET, LAS AMÉRICAS.

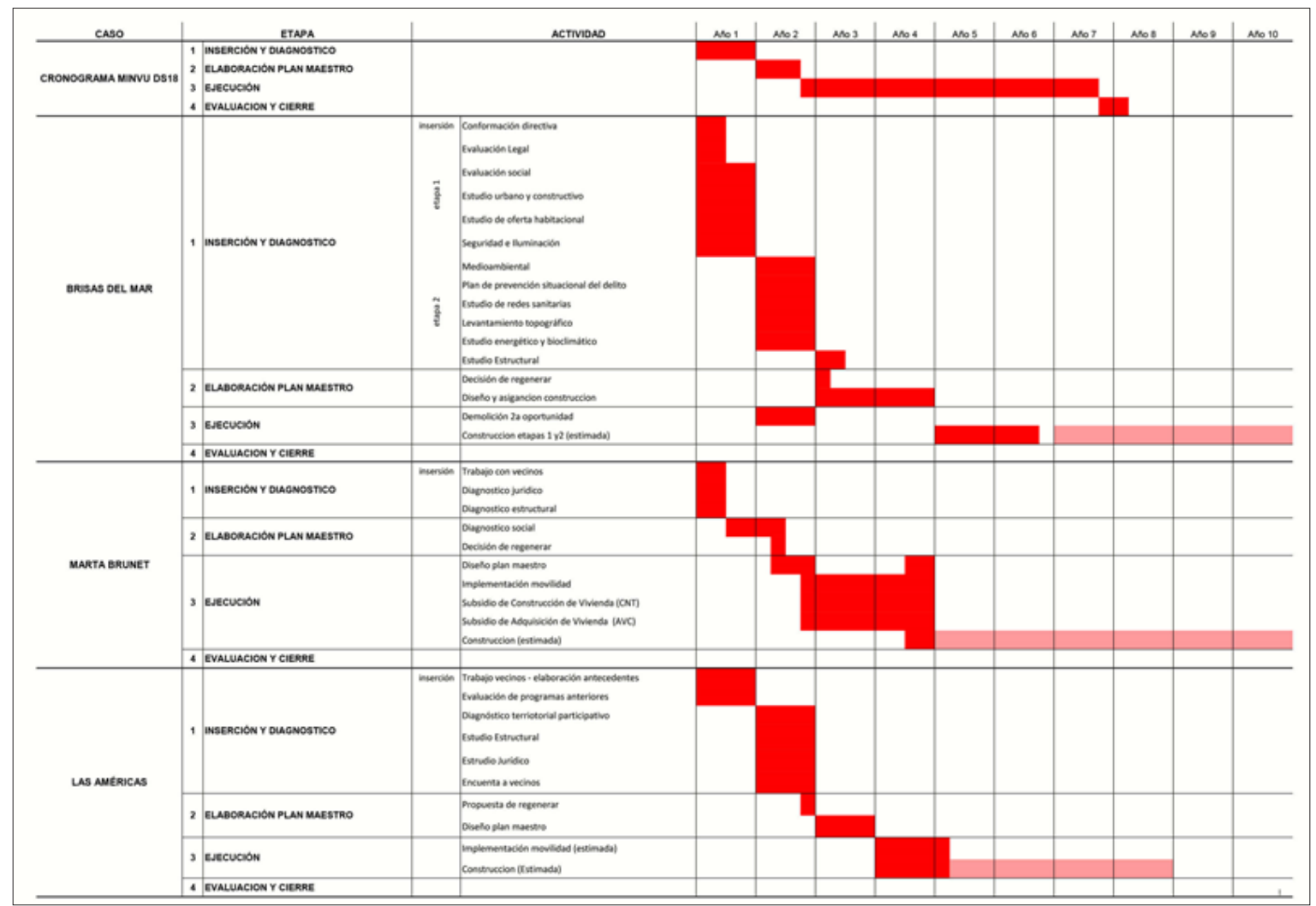

Fuente: elaboración propia.

trabajo, pérdida de redes locales de apoyo en cuidado de niños, entre otros). En ocasiones, las obras consideran intervenciones estructurales y obras de demolición y en muchos casos se hace necesario la remoción de materiales con asbestos que requieren desocupar el conjunto antes de la obra. Para proveer vivienda temporal a los residentes existen las opciones del Subsidio de Arriendo (DS-52), la implementación de viviendas prefabricadas en obra (containers) o la asignación directa de departamentos por parte del gobierno. 
TABLA 4. DIMENSIONES ESENCIALES Y COMPLEMENTARIAS.

Etapa

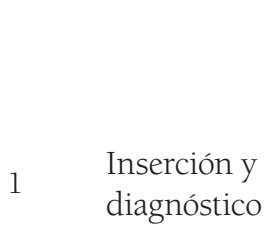

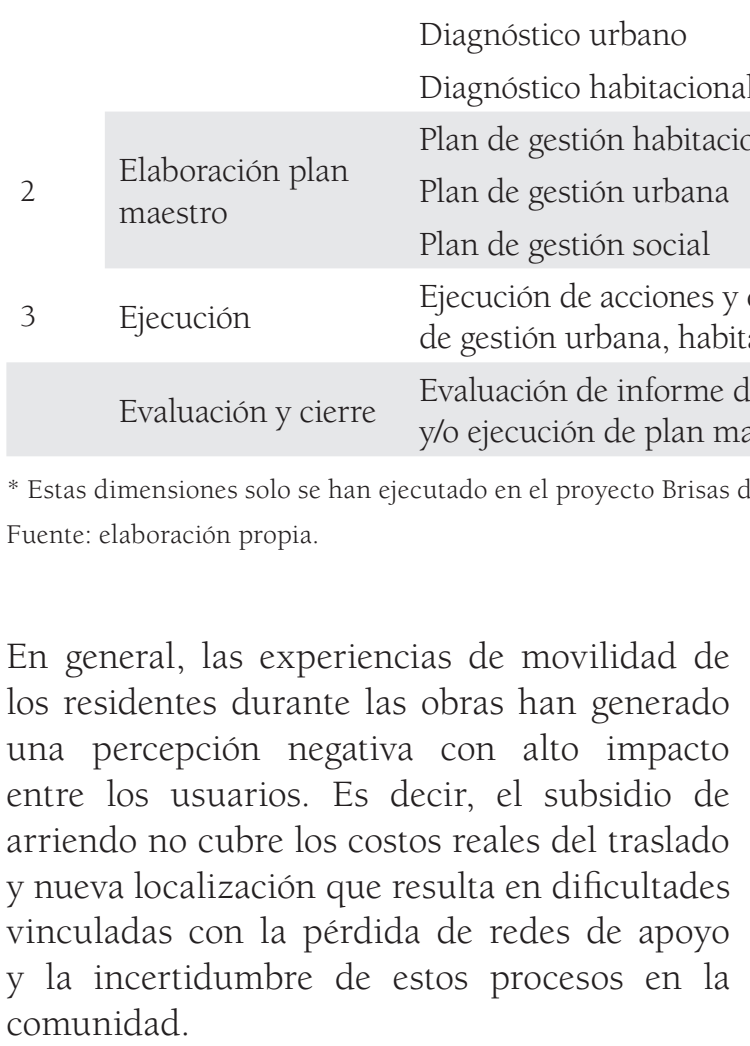

\section{Dimensiones esenciales}

Conformación Comité Regeneración

Diagnóstico legal

Diagnóstico estructural
Dimensiones complementarias

Diagnóstico seguridad vecinal *

Diagnóstico de eficiencia energética *

Diagnóstico áreas verdes y paisajismo *

Catastro de mascotas * 
van surgiendo los casos, aumentando así la incertidumbre de los vecinos.

Es importante destacar como antecedente los casos de la rehabilitación de la Unidad Vecinal Portales — dañadas por el terremoto del 2010_ donde originalmente la relocalización estaba planificada en seis meses y finalmente tard 18 meses, generando gran descontento entre los vecinos; y la rehabilitación del conjunto Volcán San José, donde los departamentos que fueron desocupados para su reparación fueron objeto de tomas ilegales por parte de familias de un campamento cercano, generando dificultades para los residentes originales y también para los encargados del proyecto.

\section{Construcción}

De todas las dimensiones involucradas en las operaciones de regeneración y rehabilitación, la construcción es la que resulta más predecible en cuanto a sus costos y plazos, por lo que su ejecución e impacto puede ser diseñado con gran precisión, disminuyendo la incertidumbre. En este sentido, es importante destacar que el uso de tecnologías y sistemas constructivos estándar con programación de faenas, plazos de ejecución y estimación de costos conocidos representa una gran ventaja y debe ser abordada al momento de plantear soluciones prefabricadas o innovaciones tecnológicas, exigiendo validaciones previas que aseguren costos, plazos y desempeños superiores a las soluciones tradicionales.

Las intervenciones realizadas en los casos de estudio consideran estructura de albañilería armada, losas de hormigón ejecutadas en obra y estructura de madera para la cubierta de acuerdo al Listado Oficial de Soluciones Constructivas MINVU. Es importante destacar que este catálogo no incluye soluciones constructivas con un alto grado de prefabricación, limitando las alternativas de intervención a la aplicación de sistemas constructivos tradicionales. Estos sistemas se denominan como "construcción húmeda" y están compuestos por estructura de hormigón armado, muros de albañilería y revoques interiores. Comúnmente, este tipo de construcción es lento, pesado y más caro (Bris, Menéndez-Pidal, Muñoz de Cuerva y Saint-Exupéry, 2013). Es un proceso artesanal que se desarrolla in-situ, queda condicionado a las capacidades de los distintos oficios y que, a veces, hasta obliga a realizar retrocesos sobre los mismos trabajos (e.g. cuando se pica una pared construida para instalar redes).

Este estudio propone que la incorporación de soluciones constructivas prefabricadas permitiría hacer más eficiente la ejecución de la obra, minimizar las externalidades negativas de las instalaciones de faenas y disminuir considerablemente los plazos de ejecución. Esta condición también reduciría el impacto en la movilidad temporal de las familias. 


\section{Enriquecer la metodología para integrar la realidad}

La clasificación de variables en dos grupos (dimensiones esenciales y complementarias) busca establecer la ruta crítica para la ejecución de los proyectos de regeneración y rehabilitación, y la superposición en el tiempo de la mayor cantidad de variables con el fin de acortar los lazos totales del proceso. El estudio de casos describe una organización cronológica y lineal de actividades donde cada partida antecede a la otra, cuando en realidad lo que se debiese buscar es el desarrollo en paralelo de la mayor cantidad de actividades posibles. Un ejemplo de actividades lineales es la relación entre los diagnósticos necesarios previa a la elaboración del plan maestro, así como el diseño arquitectónico necesario para dar inicio a las obras de construcción. Se pueden considerar como actividades paralelas la gestión simultánea del diagnóstico legal, estructural y evaluación de las instalaciones existentes. A diferencia de un modelo de gestión lineal, este estudio propone considerar la superposición en el tiempo de las actividades paralelas con el objetivo de reducir los plazos del proceso y disminuir costos.

Dentro de las dimensiones esenciales se han identificado dos operaciones capaces de disminuir el plazo total de la ejecución. a) Se recomienda la implementación del diagnóstico legal y estructural desde el inicio del proyecto, junto con el inicio del trabajo con la comunidad. Se estima que hacer este trabajo en forma paralela y no secuencial permitiría reducir los plazos en 6 meses.

b) La elección de sistemas constructivos utilizados en estos proyectos posee una incidencia directa en los plazos de ejecución y la calidad de la obra. Se recomienda realizar la gestión y desarrollo requerido para ampliar las opciones de los sistemas disponibles e incorporar alternativas prefabricadas. Estas alternativas permitirían promover procesos industriales donde se estima que la reducción de plazos de instalación de obras de montaje en seco sería entre 30\% y 50\%.

En términos sociales, el proyecto debe tener en cuenta no solo los impactos positivos generados para los residentes, sino también la incertidumbre y temor que puede suscitar entre ellos, ya que estos elementos son los que los disponen a colaborar (o resistir) el proyecto. Pensar en estrategias de comunicación y participación efectiva para todas las etapas del proyecto y en soluciones técnicas, que minimicen los aspectos más difíciles de afrontar por los vecinos, resulta esencial para la colaboración de los vecinos, la pertinencia del proyecto, y la factibilidad social del mismo. 


\section{Conclusiones}

A partir del estudio de tres casos de proyectos de regeneración y rehabilitación de vivienda colectiva en altura en Chile, el trabajo de investigación desarrollado por el equipo PlusChile y una revisión bibliográfica del estado del arte nacional e internacional, el presente estudio identifica las variables relevantes en el desarrollo de dichos procesos, establece una relación cronológica entre ellas y determina los hitos críticos en su desarrollo y ejecución, estableciendo recomendaciones para optimizar su programación.

Las líneas o ejes de acción involucrados en los procesos de regeneración y rehabilitación de conjuntos de vivienda poseen un fuerte carácter interdisciplinario, por lo que cualquier optimización, en su integración, pasa por un proceso coordinado de gestión que involucre las tres etapas principales - diagnóstico, proyecto y ejecución-, identificando la ruta crítica del proyecto para superponer la mayor cantidad de líneas de acción posibles, reduciendo así los plazos, con la consiguiente disminución de los costos asociados a la construcción y a los subsidios de arriendo y a los costos vinculados a la relocalización de las familias involucradas.
Así, la discriminación propuesta entre dimensiones esenciales y complementarias busca establecer un marco mínimo de acción, capaz de reducir la incertidumbre frente a proyectos de esta envergadura.

De forma más específica, se ha detectado que, dentro del grupo de las dimensiones esenciales, hay dos ejes de acción que tienen incidencia directa sobre la programación total, afectando sensiblemente los plazos totales de ejecución.

Por una parte, en la etapa de diagnóstico, se recomienda la aplicación temprana y simultánea del estudio legal, análisis estructural y desarrollo del trabajo comunitario. Se debe considerar que el estudio legal también puede prestar apoyo en contactar vecinos que, siendo propietarios, no se encuentren residiendo en el conjunto.

Por otra parte, en la etapa de movilidad y construcción, se ha podido establecer una relevancia significativa de los sistemas constructivos utilizados en los plazos de ejecución y de la calidad ambiental del conjunto durante el proceso de edificación. Por ejemplo, la aplicación de sistemas prefabricados podría reducir significativamente los plazos de construcción y disminuir las externalidades negativas que la misma representa para los residentes. 
Mediante la reorganización de estas actividades y la implementación de innovación tecnológica constructiva en su ejecución se podría generar un mejoramiento en los procesos de gestión. La aplicación de estas recomendaciones colaboraría a promover y masificar los proyectos de regeneración de conjuntos de vivienda social en altura en Chile. El diseño específico de estas acciones requiere de una profundización de la investigación que en el fututo incorpore la participación de entidades del gobierno, la industria privada y la academia.

Por último, es importante señalar que la idea de regeneración y rehabilitación debe ser entendida de un modo flexible, donde el objetivo final debe ser siempre la mejora en la calidad de la vivienda y de los barrios existentes, reduciendo la brecha social de la vivienda en el país.

Cada caso es diferente y presenta sus propias particularidades —localización, estado de deterioro, manejo de espacios públicos y áreas verdes, situaciones de seguridad y conflicto social, obsolescencia en las instalaciones, superficie insuficiente en la vivienda o escala de intervención. Sin embargo, el proceso de gestión para intervenirlos y mejorar sus condiciones basales puede ser guiado mediante una metodología común, capaz de articular a los diferentes actores involucrados y de reunir las dimensiones involucradas en un proceso holístico, incrementando sus posibilidades de éxito.

\section{Agradecimientos}

El equipo de investigadores quiere agradecer la buena disposición, la colaboración en este estudio y el trabajo en terreno de los equipos del MINVU y SERVIU del Programa de Regeneración de Condominios Habitacionales. En específico, Ignacio Moulian, Víctor Sepúlveda, Álvaro Poblete, Héctor Valencia, Ingrid Hidalgo, Nathalie Becerra, María Angélica Contreras, y Melissa Analoca. También se agradece a Rodrigo Olivares, ex SEREMI de Vivienda y Urbanismo en la Región del Maule, al grupo de estudiantes de magister de la Escuela de Arquitectura de la Pontificia Universidad Católica de Chile -que desde el año 2017 han contribuido al desarrollo de esta investigación-, a Thomas Batzenshlager y Paula Martínez por su trabajo en el equipo PLUS-Chile. Y de forma muy especial, a Frederic Druot por su generosa colaboración. 


\section{Referencias bibliográficas}

Assistance documents for EU member states in developing long term strategies for mobilising investment in building energy renovation. (2013). Recuperado de https://www.epbd-ca. eu/outcomes/EED-Article4-composite-document-final.pdf.

Bris, P., Menéndez-Pidal, S. Muñoz de Cuerva, F., y Saint-Exupéry, M. (2013). Construcción de viviendas mediante contenedores de obra. En Jornadas internacionales de investigación en construcción: vivienda: pasado, presente y futuro: resúmenes y actas. España: CSIC, Instituto Eduardo Torroja.

Casanovas, X., Cuchí, A., Mas Herrero, J., y Rubio del Val, J. (2018). Por un cambio en las politicas públicas de fomento de la rehabilitación residencial: los municipios, pieza clave en un marco de cooperación institucional. España: GTR (Grupo de Trabajo sobre Rehabilitación). Recuperado de http://www.conama2018.org/download/ bancorecursos/C2018/Informe\%20GTR\%20 Ciudades.pdf

Cuchí, A. y Sweatman, P. (2013). Informe GTR 2014 estrategia para la rehabilitación claves para transformar el sector de la edificación en España. España: GTR (Grupo de Trabajo sobre Rehabilitación). Recuperado de https://www.miteco. gob.es/images/es/rciinformegtr2014 tcm30178967.pdf
D'Oca, S. y Veld, P. (2018). People-centered deep renovation practices: from challenges to strategies. TEMA Technologies Engineering Materials Architecture, 4(3) 81-91. https://doi. org/10.17410/tema.v4i3.206

Dall'0, G., Galante, A., y Pasetti, G. (2012). A methodology for evaluating the potential energy savings of retrofitting residential building stocks. Sustainable Cities and Society, 4, 12-21. https://doi.org/10.1016/j.scs.2012.01.004

Decreto 18. Reglamento del programa de regeneración de conjuntos habitacionales de viviendas sociales, Santiago, Chile, 23 de marzo de 2018.

Druot, F., Lacaton, A. y Vassal, J. P. (2007). Plus - les grands ensembles de logements, territoires d'exception. Barcelona: Gustavo Gili.

Ferrante, A. y Semprini, G. (2011). Building energy retrofitting in urban areas. Procedia Engineering, 21, 968-975. https://doi.org/10.1016/j. proeng.2011.11.2101

Fotopoulou, A., Semprini, G., Cattani, E., Schihin, Y., Weyer, J., Gulli, R., y Ferrante, A. (2018). Deep renovation in existing residential buildings through façade additions: A case study in a typical residential building of the 70s. Energy and Buildings, 166, 258-270. https://doi.org/10.1016/j.enbuild.2018.01.056

Ibañez, I. (2015). Insatisfacción barrial en conjuntos de viviendas sociales. ÉnfaCIS (8). 
IDIEM. (2014). Investigación desarrollo e innovación de estructura y materiales, estudio de habitabilidad y evaluación estructural de condominio "Marta Brunet” - región metropolitana. Santiago, Chile: Facultad de Ingeniería de la Universidad de Chile.

Lavanchy, J. L. (2020). El hacinamiento en los conjuntos de bloques C. Definir las variables que influyen sobre el fenómeno para responder a la problemática. (Tesis magister en arquitectura, sin publicar). Pontificia Universidad Católica de Chile, Santiago, Chile.

Mikulic, D., Rasic Bakaric, I., y Slijepcevic, S. (2016). The economic impact of energy saving retrofits of residential and public buildings in Croatia. Energy Policy, 96, 630-644. https:// doi.org/10.1016/j.enpol.2016.06.040

Miloni, R., Grischott, N., y Zimmermann, M. (2011). Building renovation case studies. Switzerland: Empa, Building Science and Technology Lab.

Ministerio de Vivienda y Urbanismo. (2014a). Vivienda social en copropiedad. Catastro nacional de condominios habitacionales. Santiago: Autor.

Ministerio de Vivienda y Urbanismo. (2014b). Vivienda social en copropiedad. Memoria de tipologías en condominios sociales. Santiago: Autor.

Monge-Barrio, A. y Sánchez-0stiz, A. (2015). Energy efficiency and thermal behaviour of attached sunspaces, in the residential architecture in Spain. Summer conditions. Energy and Buildings, 108, 244-256. https://doi.org/10.1016/j. enbuild.2015.09.037

Paiho, S., Ketomaki, J., Kannari, L., Hakkinen, T., y Shameikka, J. (2019). A new procedure for assessing the energy-efficient refurbishment of buildings on district scale. Sustainable Cities and Society, 46, 101454. https://doi.org/10.1016/j.scs.2019.101454

Rodríguez, A. y Sugranyes, A. (2005). Los con techo. Un desafio para la política de vivienda social. Santiago, Chile: SUR.

Salcedo, R., Hermansen, P., y Rasse, A. (2017). Habitando el gueto. Estrategias para sobrevivir un espacio público deteriorado: El caso de Bajos de Mena en Santiago, Chile. En T. Errázuriz y R. Greene (Eds.) Salcedo. Talca, Chile: Bifurcaciones.

Salcedo, R., Rasse, A., y Cortés, A. (2013). Del proyecto colectivo al aislamiento: la desaparición de la noción de barrio. En F. Sabatini, G. Wormald y A. Rasse (Eds.), Segregación de la vivienda social: Ocho conjuntos en Santiago, Concepción y Talca. Santiago, Chile: Instituto de Estudios Urbanos y Territoriales, Pontificia Universidad Católica.

Soto, V. (2020). Desde la infraestructura hacia la estructura, programa y forma. (Tesis magister en arquitectura, sin publicar). Pontificia Universidad Católica de Chile, Santiago, Chile. 
Tirado Herrero., S., Jiménez Meneses, L., López Fernández, J.L., y Martín García, J. (2014).

Pobreza energética en España. Análisis de tendencias. Madrid: Asociación de Ciencias Ambientales.

United Nations. (2015). World population prospects: The 2015 revision, key findings and advance tables (Working paper No. ESA/P/WP.241).

Villagra, E. (2018). Regeneración conjuntos habitacionales (Informe práctica profesional, sin publicar). Universidad de Chile, Santiago, Chile. 


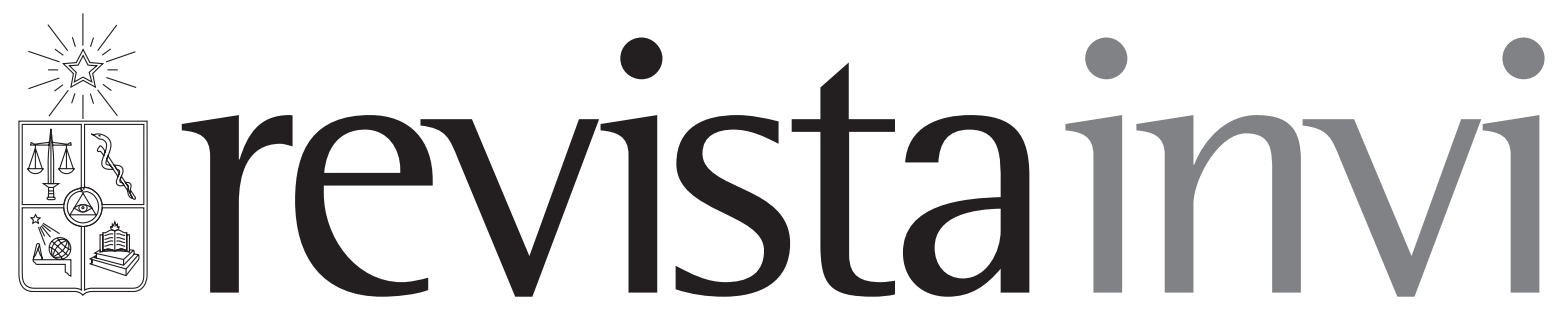

Revista INVI es una publicación periódica, editada por el Instituto de la Vivienda de la Facultad de Arquitectura y Urbanismo de la Universidad de Chile, creada en 1986 con el nombre de Boletín INVI. Es una revista académica con cobertura internacional que difunde los avances en el conocimiento sobre la vivienda, el hábitat residencial, los modos de vida y los estudios territoriales. Revista INVI publica contribuciones originales en español, inglés y portugués, privilegiando aquellas que proponen enfoques inter y multidisciplinares y que son resultado de investigaciones con financiamiento y patrocinio institucional. Se busca, con ello, contribuir al desarrollo del conocimiento científico sobre la vivienda, el hábitat y el territorio y aportar al debate público con publicaciones del más alto nivel académico.

Director: Dr. Ricardo Tapia Zarricueta, Universidad de Chile, Chile.

Editor: Dr. Luis Campos Medina, Universidad de Chile, Chile.

Editor asistente: Dr. Walter Imilan, Universidad de Chile, Chile.

Coeditora: Srta. Sandra Rivera, Universidad de Chile, Chile.

\section{COMITÉ EDITORIAL:}

Dr. Victor Delgadillo, Universidad Autónoma de la Ciudad de México, México.

Dra. María Mercedes Di Virgilio, CONICET/ IIGG, Universidad de Buenos Aires, Argentina.

Dra. Irene Molina, Uppsala Universitet, Suecia.

Dr. Gonzalo Lautaro Ojeda Ledesma, Universidad de Valparaíso, Chile.

Dra. Suzana Pasternak, Universidade de São Paulo, Brasil.

Dr. Javier Ruiz Sánchez, Universidad Politécnica de Madrid, España.

Dra. Elke Schlack Fuhrmann, Pontificia Universidad Católica de Chile, Chile.

Dr. Carlos Alberto Torres Tovar, Universidad Nacional de Colombia, Colombia.

Sitio web: http://www.revistainvi.uchile.cl/

Correo electrónico: revistainvi@uchilefau.cl

Licencia de este artículo: Creative Commons Atribución-Compartirlgual 4.0 Internacional (CC BY-SA 4.0) 